\title{
The impact on human visual performance when viewing 2-D and 3-D movies
}

\author{
Fan Yang ${ }^{\mathrm{a}, \mathrm{b}, \mathrm{c}, *}$, Huiru Gu${ }^{\mathrm{d}, \mathrm{e}, \mathrm{f}}$, Man Li ${ }^{\mathrm{d}, \mathrm{e}, \mathrm{f}}$, Jianqi Cai ${ }^{\mathrm{g}}$, Qianxiang Zhou ${ }^{\mathrm{a}, \mathrm{b}}$ and Wing-Kai Lam ${ }^{\mathrm{c}}$ \\ ${ }^{a}$ School of Biological Science and Medical Engineering, Beihang University, Beijing, China \\ ${ }^{\mathrm{b}}$ Beijing Advanced Innovation Centre for Biomedical Engineering, Beihang University, Beijing, China \\ ${ }^{\mathrm{c}}$ Li Ning Sports Science Research Center, Beijing, China \\ ${ }^{\mathrm{d}}$ National Research Center for Rehabilitation Technical Aids, Beijing, China \\ ${ }^{\mathrm{e}}$ Beijing Key Laboratory of Rehabilitation Technical Aids for Old-Age Disability, Beijing, China \\ ${ }_{\mathrm{f}}^{\mathrm{f}}$ Key Laboratory of Rehabilitation Technical Aids Analysis and Identification of the Ministry of Civil \\ Affairs, Beijing, China \\ ${ }^{\mathrm{g}}$ China National Institute of Standardization, Beijing, China
}

\begin{abstract}
.
BACKGROUND AND OBJECTIVE: To examine the human visual performance (wavefront aberration) and subjective questionnaire (SQ) of visual fatigue when viewing 2-D and 3-D movies.

METHODS: Thirty healthy adults observed 2-D and 3-D movies on the same television from a 3m distances during 2D, 3D-A (with better 3D glasses), and 3D-B (with poorer 3D glasses) viewing conditions. Visual quality index, including modulation transfer function index (MTFI), higher order aberration root mean square (RMS), vertical coma (VC), horizontal coma (HC) and spherical aberration (SA), were assessed before and after each viewing condition. One-way repeated measures ANOVA was performed to assess the changes of each test variable before and after movie viewing.

RESULTS: Participants watching movies with 3D-B conditions experienced higher change of MTFI, RMS, VC and HC but smaller SQ, compared with 2D and 3D-A $(P<0.05)$. Additionally, higher MTFI but smaller SQ was found for 3D-A compared with $2 \mathrm{D}$ viewing condition $(P<0.05)$.

CONCLUSIONS: While prolonged viewing 2-D and 3-D movies would lead to poorer visual performance, 3-D glasses with better quality can play the major role in reducing visual ability for users. The change of human eye wavefront aberration might be useful for the evaluation of visual fatigue in the future.
\end{abstract}

Keywords: 2-D/3-D, wavefront aberration, visual function, visual fatigue

\section{Introduction}

Television (TV) is one of the most influential media for popular entertainment. As rapid development of high quality of TV content, people, particularly for children, spend more and more time on TV [1]. Recent research suggested that viewing of three-dimensional (3-D) movies is rapidly increasing for better quality of life and would be of higher prevalence, compared with the traditional two-dimensional (2-D) TV and movies [2]. However, it has been concerned that prolonged viewing 3-D TV would be the

\footnotetext{
${ }^{*}$ Corresponding author: Fan Yang, School of Biological Science and Medical Engineering, Beihang University, Beijing, China. Tel.: +86 18911326682; E-mail: yzyangfan@ foxmail.com.
}

0928-7329/18/\$35.00 (c) 2018 - IOS Press and the authors. All rights reserved

This article is published online with Open Access and distributed under the terms of the Creative Commons Attribution NonCommercial License (CC BY-NC 4.0). 
risk factor of damaging the human visual performance [3]. The authors argued that visual information provided by a 3-D stereoscopic TV introduced the disparity between the overlapped images that allow objects to be located perceptually in front of or behind screen (3-D visual effect) [3]. This may affect the regular visual processing when using in the real world. Therefore, it is important to evaluate the associated effect between 2-D and 3-D displays.

Previous studies suggested that the visual fatigue would alter the regulation of the human eye accommodation response (AC) [4]. Another study developed a questionnaire which can effectively classify the 3D motion images in accordance to subjective visual fatigue using five perception variables: (1) Eye strain, (2) general discomfort, (3) nausea, (4) Focusing difficulty and (5) headache, which were effective for classifying 3D motion images [5]. Ntuen et al. [6] examined the effect of viewing field and illumination on visual performance between 2-D and 3-D autostereoscopic displays. They found higher visual fatigue was complained when viewing 3-D compared with the 2-D counterpart. Additionally, Mun et al. [7] identified 3-D images would be of higher cognitive fatigue, as evident by higher steady-state visually evoked potential (SSVEP) and event-related potential (ERP). Furthermore, prolonged viewing 3-D TV has been suggested to be associated with dizzy, nausea, and visual fatigue [8-10]. However, most Electroencephalogram (EEG) studies on visual health have required sophisticated laboratory equipment, long data acquisition time, high signal artifact and induced discomfort. Therefore, it is important to develop reliable and accurate visual health and fatigue assessment protocols for $3 \mathrm{D}$ displays and related content.

Waveform aberration is the optical imperfections of the eye that prevent light from focus perfectly on the retina, resulting in defects in the visual image. Measuring the orthogonal Zernike polynomial expansion (wavefront technology), which describe eye pupil area optical aberration [11,12]. In addition, highorder aberration changes are vital for eye visual quality [13]. Furthermore, modulation transfer function (MTF) that is determined by the wavefront aberration can effectively reflect the overall response of space image of human eye [14]. Therefore, the purpose of this study was to evaluate the change of wavefront aberration and subjective perception before and after viewing 2-D and 3-D movies. Additionally, this study was to find the influence of visual performance by wearing different 3D glasses. This information would provide insights of the changes of human visual performance associated with 3-D movies.

\section{Materials and methods}

\subsection{Participants}

Thirty healthy adults (15 males and 15 females, mean age: $31.4 \pm 7.1$ years, myopic degree: $0-200 \mathrm{D}$ ), participated in this study. None of the participants reported of any record of cardiovascular disease or neurological disorder and did not have any drugs affecting mental pressure and visual function [15]. All participants were reported to have excellent sleep quality before the testing day. Participants were required to have normal stereoscopic feelings and sign an informed consent prior to the study. The testing protocol was approved by the Institutional Ethics Committee.

\subsection{Experimental procedure}

The participants observed the identical movies at the constant distance of 3 meter in one 2-D and two 3-D viewing sessions over the three consecutive weekends (Fig. 1a). During the 2-D viewing session (2D), participants watched the movies without glasses. During the 3-D viewing sessions (3D-A 
Table 1

Manufacturing performance of the tested 3-D glasses

\begin{tabular}{lcccccc}
\hline & \multicolumn{2}{c}{ 3D-A } & & \multicolumn{2}{c}{ 3D-B } & National standard range \\
\cline { 2 - 3 } & Measured value & Evaluation & & Measured value & Evaluation & \\
\hline Polarizing efficiency & $99.9 \%$ & Passed & & $98.8 \%$ & Failed & $\geqslant 99.9 \%$ \\
Phase difference $(\mathrm{nm})$ & $125-127$ & Passed & & $109-112$ & Failed & $125 \pm 5$ \\
Joint angle $\left(^{\circ}\right)$ & 45.6 & Passed & & 46.1 & Qualified & $45.0 \pm 1.5$ \\
\hline
\end{tabular}

Note: The national standard of 3D-A pasted standard requirement was adopted from QB/T 4735-2014 Polarized 3D glasses (in Chinese) [16].

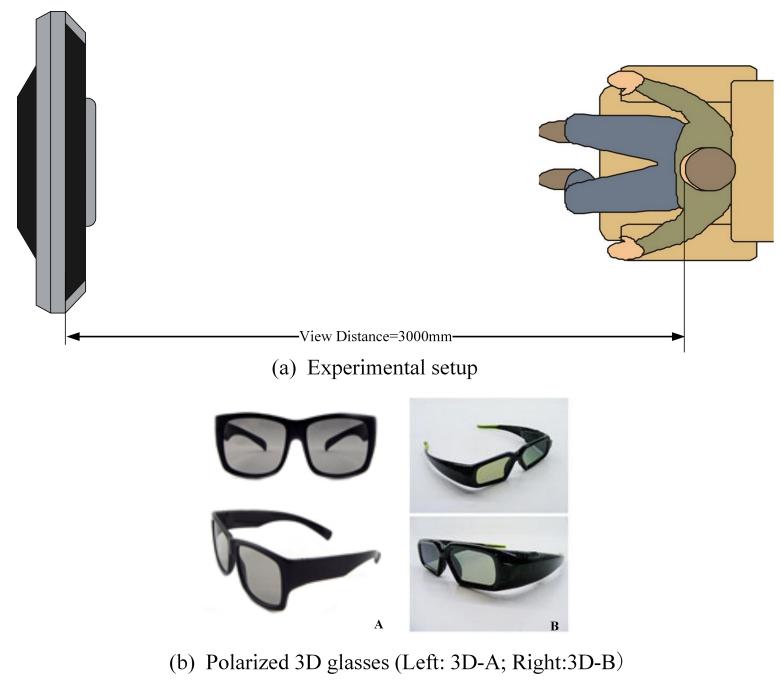

Fig. 1. a) Experimental setup and b) Polarized 3D glasses (left: 3D-A; right: 3D-B).

and 3D-B), participants watched the movies when wearing two different types of 3-D polarized glasses (Fig. 1b). The manufacturing performance of 3D-A (P3D-1, PROSUN, China) and 3D-B (P3D-2, PROSUN, China) are provided in Table 1. The same movie "As The Light Goes Out" (Emperor Entertainment Group Limited, Mandarin, 2014) was presented in all viewing conditions (2D, 3D-A and 3D-B). The participants were informed to focus on the movie only during each viewing session. The 2-D/3-D movies were displayed on a 42 inch LED full HD television (LG 3D TV type 42LW5500-CA, LG Electronics Inc., Korea), with a resolution ratio of $1920 \times 1080$ and frequency of $100 \mathrm{~Hz}$. It utilized polarized light (and using polarized 3D glasses) to create stereoscopic depth (i.e., 3D images). The identical protocol and movie were carried out with one-week interval for the subsequent viewing sessions. All viewing sessions were randomly assigned across participants.

Wavefront aberrations of participants were measured with Nidek OPD-Scan (Nidek, Gamagori, Japan) aberrometer before and after each of the viewing conditions (2D, 3D-A, and 3D-B). Additionally, the participants provided their perception of eye fatigue level at the end of each viewing condition (Table 2).

\subsection{Data processing}

The screenshots of visual performance are shown in Fig. 2. Modulation Transfer Function (MTF), higher order Root-Mean-Square (RMS), Vertical Coma (VC), Horizontal Coma (HC), and Spherical Aberration (SA) were determined and before and after viewing movies. 
Table 2

Eye fatigue scales (subjective questionnaire, SQ)

\begin{tabular}{cll}
\hline Level & Fatigue level & Symptom \\
\hline 0 & No fatigue & Without obvious characteristics \\
1 & Mild fatigue & Mild eyestain and gritty \\
2 & Less moderate fatigue & Less moderate eyestrain and dizziness \\
3 & Moderate fatigue & Moderate eyestrain, stinging, dizzy and headache \\
4 & Less severe fatigue & Less severe eyestrain, gritty, dizziness and nausea \\
5 & Severe fatigue & Severe eyestrain, headache, nausea and vomiting \\
\hline
\end{tabular}

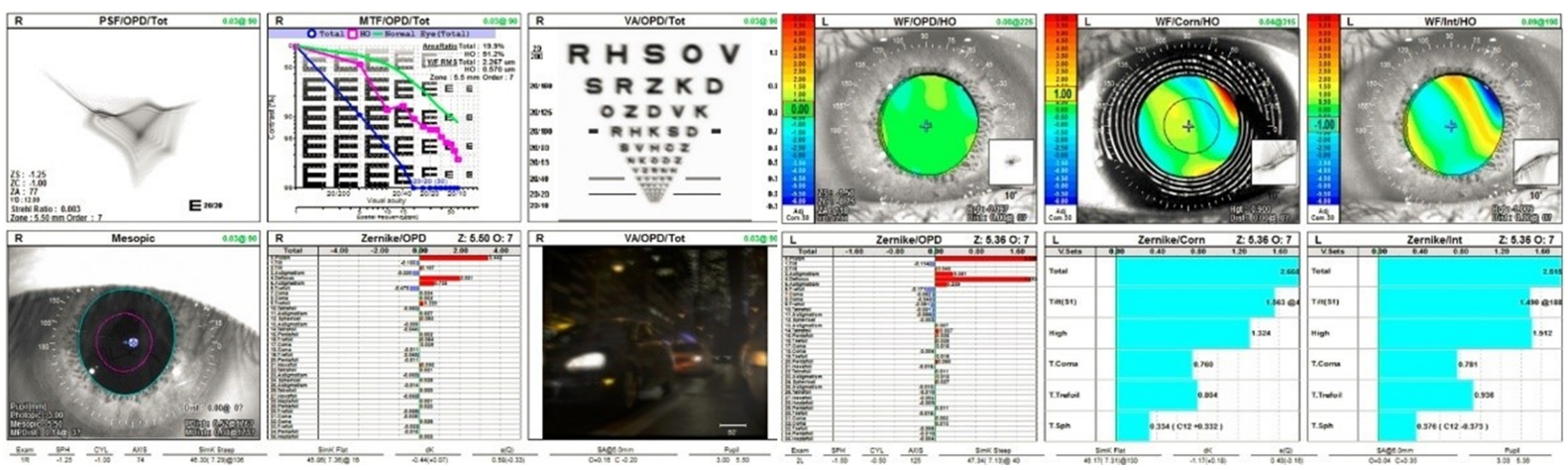

Fig. 2. The screenshots of visual quality performance.

\subsubsection{Modulation transfer function (MTF)}

MTF was the most important and fundamental evaluation method in visual performance. MTF denoted the transmission of different spatial frequencies (i.e. Optical transfer function, (OTF)). OTF was the Fourier transform of the Point Spread Function (PSF):

$$
\operatorname{OTF}(\varepsilon, \eta)=\operatorname{MTF}(\varepsilon, \eta) e^{-\mathrm{iPTF}(\varepsilon, \eta)}
$$

where $\varepsilon$ and $\eta$ are the spatial frequencies in the $x$ and $y$ directions.

In this study, MTF index (MTFI) was calculated by the integral of all the MTF values within cutoff frequency (spatial frequency when MTF $=0.01)$. The absolute change of MTFI $\left(\mathrm{MTFI}_{c}\right)$ was computed the difference before and after viewing movies.

\subsubsection{Higher order root-mean-square (RMS)}

RMS accurately reflected the dimension of each order wavefront aberration [14]. RMS of higher-order aberration $\left(\sigma_{\text {high }}\right)$ were often used to evaluate the good or bad of visual performance [17]. The RMS $\sigma_{\text {high }}$ was calculated by:

$$
\operatorname{RMS} \sigma_{\text {high }}=\sqrt{\left(Z_{3}^{-3}\right)^{2}+\left(Z_{3}^{-1}\right)^{2}+\left(Z_{n}^{m}\right)^{2}}
$$

In this study, the absolute change of RMS $3^{\text {rd }}-\mathrm{RMS} 7^{\text {th }}$ and the absolute change of $\mathrm{RMS}_{a f t e r}$ and $\mathrm{RMS}_{\text {before }}\left(\mathrm{RMS}_{c}\right)$ were computed before and after viewing movies.

\subsubsection{Vertical coma $(V C)$, horizontal coma $(H C)$ and spherical aberration (SA)}

Three Zerniker coefficient of Wavefront aberrations were selected for vision correction [18], as follows:

$$
\text { Vertical Coma (VC): } Z_{3}^{-1}=\sqrt{8}\left(3 \rho^{3}-2 \rho\right) \sin \theta
$$


Table 3

RMS variables by three viewing conditions $(\mu \mathrm{m}$, mean $\pm \mathrm{SD})$

\begin{tabular}{cccccc}
\hline & RMS 3 $^{\text {rd }}$ & RMS 4 $^{\text {th }}$ & RMS $^{\text {th }}$ & RMS 6 $^{\text {th }}$ & RMS 7 $^{\text {th }}$ \\
\hline 2D & $-0.060 \pm 0.012$ & $-0.062 \pm 0.057$ & $-0.024 \pm 0.034$ & $-0.002 \pm 0.025$ & $-0.005 \pm 0.012$ \\
3D-A & $-0.077 \pm 0.093$ & $-0.060 \pm 0.070$ & $-0.027 \pm 0.023$ & $-0.008 \pm 0.016$ & $-0.005 \pm 0.010$ \\
3D-B & $-0.082 \pm 0.078^{\mathrm{ab}}$ & $-0.080 \pm 0.079^{\mathrm{ab}}$ & $-0.026 \pm 0.034$ & $-0.016 \pm 0.024$ & $-0.008 \pm 0.011$ \\
$F$ & 2.930 & 4.043 & 0.517 & 2.683 & 1.186 \\
$P$ & $<0.05$ & $<0.05$ & $>0.05$ & $>0.05$ & $>0.05$ \\
\hline
\end{tabular}

Note: "a" and "b" denoted significant differences from 2D and 3D-A, respectively $(P<0.05)$.

Table 4

Vertical Coma (VC), Horizontal Coma (HC) and Spherical Aberration (SA) by three viewing conditions ( $\mu \mathrm{m}, \mathrm{mean} \pm \mathrm{SD})$

\begin{tabular}{cccc}
\hline & VC & HC & SA \\
\hline 2D & $-0.017 \pm 0.425$ & $-0.036 \pm 0.037$ & $-0.051 \pm 0.065$ \\
3D-A & $-0.020 \pm 0.019^{\mathrm{a}}$ & $-0.038 \pm 0.071$ & $-0.052 \pm 0.059$ \\
3D-B & $-0.032 \pm 0.029^{\mathrm{ab}}$ & $-0.054 \pm 0.090^{\mathrm{ab}}$ & $-0.055 \pm 0.052$ \\
$F$ & 5.205 & 3.541 & 0.176 \\
$P$ & $<0.05$ & $<0.05$ & $>0.05$
\end{tabular}

Note: "a" and "b" denoted significant differences from 2D and 3D-A, respectively $(P<0.05)$.

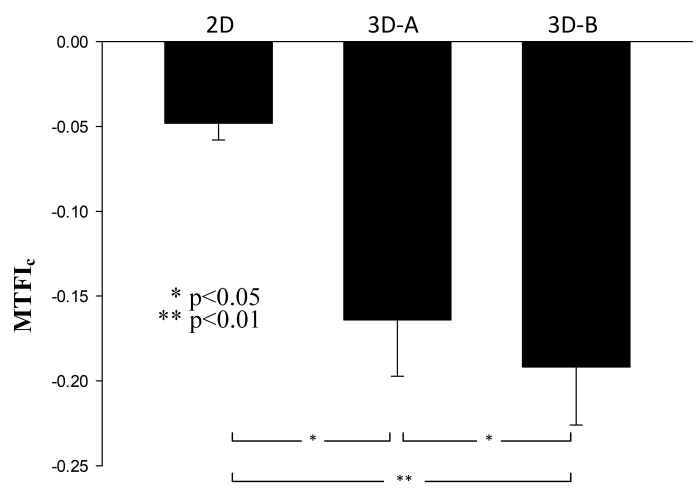

Fig. 3. Mean $\mathrm{MTFI}_{c}$ by three viewing conditions.

Horizontal Coma(HC): $Z_{3}^{1}=\sqrt{8}\left(3 \rho^{3}-2 \rho\right) \cos \theta$

Spherical Aberration(SA): $Z_{4}^{0}=\sqrt{5}\left(6 \rho^{4}-6 \rho^{2}+1\right)$

\subsubsection{Statistical analysis}

All statistical analyses were performed using SPSS 18 (IBM Corp., Armonk, NY, USA). Descriptive statistics (means and standard deviation) of wavefront aberrations and perception variables were computed across viewing conditions. A one-way repeated measures ANOVA was performed to examine if there were any significant differences on all variables. LSD post-hoc 2-tailed comparisons were computed using a criterion alpha at 0.05 .

\section{Result}

\subsection{Modulation transfer function index (MTFI)}

The one-way ANOVA analysis indicated a significant difference of $\mathrm{MTFI}_{c}$ among 2D, 3D-A, and 3D- 


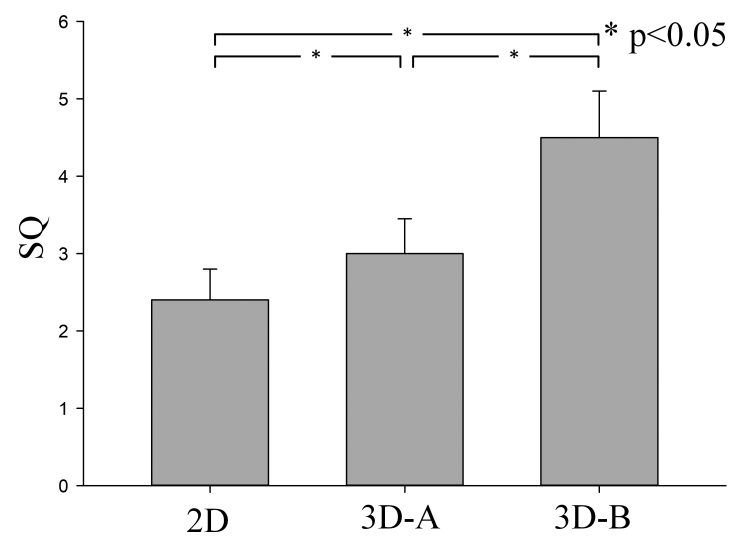

Fig. 4. Mean SQ by three viewing conditions.

B conditions ( $F=3.315, P=0.031$, Fig. 3 ). A pairwise comparison revealed that significant smaller $\mathrm{MTFI}_{c}$ were found for $2 \mathrm{D}$ compared with 3D-A $(P<0.05)$ and 3D-B $(P<0.01)$ viewing conditions. Furthermore, 3D-B had larger $\mathrm{MTFI}_{c}$ than 3D-A $(P<0.05)$.

\subsection{Higher order aberration - root mean square (RMS)}

The ANOVA analysis indicated significant main effects for RMS $3^{\text {rd }}$ and $4^{\text {th }}(P<0.05$, Table 3$)$. A pairwise comparison revealed that participants watching movie in 3D-B condition had significant larger change of RMS (i.e. RMS $3^{\text {rd }}$ and $\left.4^{\text {th }}\right)$ compared with 2D and 3D-A $(P<0.05)$.

\subsection{Vertical coma (VC), horizontal coma (HC) and spherical aberration (SA)}

The ANOVA analysis indicated significant main effects for VC $(F=5.205, P<0.05)$ and $\mathrm{HC}$ $(F=3.541, P<0.05$, Table 4$)$. A pairwise comparison revealed participants watching movie in 3D-B condition had significant larger VC and HC compared with 2D and 3D-A $(P<0.05)$. Furthermore, 3D-A had larger VC than 2D $(P<0.05)$.

\subsection{Eye fatigue score $(S Q)$}

The one-way ANOVA analysis indicated a significant difference of SQ among 2D, 3D-A, and 3D-B conditions $(F=6.71, P<0.05$, Fig. 4). A pairwise comparison revealed that significant smaller SQ were found for $2 \mathrm{D}$ compared with 3D-A and 3D-B viewing conditions $(P<0.05)$. Furthermore, 3D-B had larger SQ than 3D-A $(P<0.05)$.

\section{Conclusions}

The present study examined the human eye visual index (wavefront aberration) and subjective score after watching the same movie in three viewing conditions (2D, 3D-A and 3D-B). Overall, our findings indicated that prolonged viewing 3-D movies caused to larger change of MTFI, RMS, VC, and HC of wavefront aberration and poorer fatigue score, compared to the 2-D viewing condition. This supports the hypothesis that prolonged viewing 3-D movies would be of higher risk of damaging the human 
visual ability [19]. Recent studies on 3-D movies/images [20,21] has suggested prolonged viewing 3-D television could also cause visual fatigue, brain fatigue, change of pulse and increased of heart rate. Therefore, the applications of 3-D imaging technology could be of concerns to the health issue in the future.

It is suggested that high quality 3D glasses could be high importance to protect the human eyes when watching television in 3-D as their daily entertainment. In our study, the glasses used in 3D-A had better optical qualities (qualified polarizing efficiency and phase difference) than the glasses used in 3D-B viewing session and the present results showed that better qualified 3-D glasses can relief subjective visual fatigue (i.e. lower SQ score) and remain better visual performance (i.e. MTFI, VC, RMS $3^{\text {rd }}$ and RMS $\left.4^{\text {th }}\right)$. This is in line with the study of Wenzel et al. [22], which indicated that wearing different shutter 3D glasses can cause influence users' EEG. These data suggest that good quality control of 3D glasses at the manufacturing process would be helpful and effective to enhance the visual health.

Several wavefront aberration derived variables were selected in this study, as they were considered as reliable indicators of vision performance and fatigue. Modulation transfer function (MTF) and Zernike polynomials (VC, HC and SA, Table 4) index can effectively evaluate the effectiveness of the vision performance rehabilitation/training after refractive surgery [23,24]. The present study found that a decreased change in MTFI was found after prolonged movies viewing. Moreover, watching 3-D movies would have greater impact on visual performance compared (MTFI, VC and HC) with 2-D movies. RMS of higher order aberrations is another commonly used index of visual ability [25]. The current study found significant difference only exist in two higher order aberration variables (RMS3 ${ }^{\text {rd }}$ and RMS4 ${ }^{\text {th }}$ ) but not exist for other aberration variables (RMS $5^{\text {th }}$ to $7^{\text {th }}$ ) between 2-D and 3-D viewing conditions. It seems the RMS variables would be of inferior discriminating power than the MTFI variable for the evaluation and diagnosis of visual performance. Further research on the relationship among subjective visual score, MTFI, Zernike polynomials, and RMS of higher order aberrations is necessary. The limitation of this study is that it primarily focused on the difference between 2-D and 3-D viewing conditions, and did not investigate how 3-D content may lead to visual fatigue. In the future, more research needs to be done on the mechanism of visual fatigue from 3-D content, in order to offer more practical advice to 3-D companies and also protect the visual health of consumers.

In summary, the visual stimulation of 2-D and 3-D movies can influence human visual function after prolonged viewing. Compared to 2-D movies, 3-D movies would be more susceptible to visual fatigue. Better quality of 3-D glasses can play the major role in reducing visual fatigue for users. MTFI and coma aberration in human wavefront aberration variables might provide additional insights to assess the level of human visual fatigue in the future.

\section{Acknowledgments}

This research was funded by Electronic Information Equipment System Research of Key Laboratory of Basic Research Projects of National Defense Technology (DXZT-JC-ZZ-2015-016) and reform and development funds of Beijing Municipal Institute of Labor Protection.

\section{Conflict of interest}

None to report. 


\section{References}

[1] Tudor-Locke C, Ainsworth BE, Adair LS, et al. Physical activity and inactivity in Chinese school-aged youth: The China Health and Nutrition Survey. International Journal of Obesity 2003; 27(9): 1093-1099. DOI: 10.1038/sj.ijo.0802377

[2] Kunic S, Sego Z. 3D television ELMAR. Proceedings IEEE 2011; 127-131.

[3] Maydeo AP, Bhandari S, Bapat M, et al. Potential hazards of viewing 3-D stereoscopic television, cinema and computer games: A review. Ophthalmic Physiol Opt 2011; 31(2): 111-122. DOI: 10.1111/j.1475-1313.2011.00822.x

[4] Yano S, Ide S, Mitsuhashi T, et al. A study of visual fatigue and visual comfort for 3D HDTV/HDTV images. Displays 2002; 23(4): 191-201. DOI: 10.1016/S0141-9382(02)00038-0

[5] Kuze J, Ukai K. Subjective evaluation of visual fatigue caused by motion images. Displays 2008; 29(2): 159-166. DOI: 10.1016/j.displa.2007.09.007

[6] Ntuen CA, Goings M, Reddin M, et al. Comparison between 2-D \& 3-D using an autostereoscopic display: The effects of viewing field and illumination on performance and visual fatigue. International Journal of Industrial Ergonomics 2009; 39(2): 388-395. DOI: 10.1016/j.ergon.2008.07.001

[7] Mun S, Park MC, Park S, et al. SSVEP and ERP measurement of cognitive fatigue caused by stereoscopic 3D. Neuroscience Letters 2012; 525(2): 89. DOI: 10.1016/j.neulet.2012.07.049

[8] Ukai K, Howarth PA. Visual fatigue caused by viewing stereoscopic motion images: Background, theories, and observations. Displays 2008; 29(2): 106-116. DOI: 10.1016/j.displa.2007.09.004

[9] Lambooij M, Fortuin M, Ijsselsteijn W, et al. Measuring visual discomfort associated with 3D displays. International Society for Optics and Photonics 2009; 931-943.

[10] Lambooij M, Ijsselsteijn WA, Heynderickx I. Visual discomfort of 3D TV: Assessment methods and modeling. Displays 2011; 32(4): 209-218. DOI: 10.1016/j.displa.2011.05.012

[11] Maeda N. Wavefront technology in ophthalmology. Current Opinion in Ophthalmology 2001; 12(4): 294. DOI: 10.1097/00055735-200108000-00009

[12] Thibos LN, Applegate RA, Schwiegerling JT, et al. Standards for reporting the optical aberrations of eyes. Journal of Refractive Surgery 2002; 18(5): S652.

[13] Howland HC. High order wave aberration of eyes. Ophthalmic \& Physiological Optics the Journal of the British College of Ophthalmic Opticians 2002; 22(5): 434-439. DOI: 10.1046/j.1475-1313.2002.00072.x

[14] Cheng X, Thibos LN, Bradley A. Estimating visual quality from wavefront aberration measurements. Journal of Refractive Surgery 2003; 19(5): S579-S584.

[15] Craig A, Hancock K, Craig M. The lifestyle appraisal questionnaire: A comprehensive assessment of health and stress. Psychology \& Health 1996; 11(3): 331-343. DOI: 10.1080/08870449608400262

[16] QB/T 4735-2014, Polarized 3D galsses (in Chinese).

[17] Cheng AC, Rao SK, Lam DS. Ocular higher-order aberrations in eyes with supernormal vision. American Journal of Ophthalmology 2005; 140(5): 225-228. DOI: 10.1016/j.ajo.2004.08.035

[18] JimÉnez JÉR, Castro JÉJ, JimÉnez R, et al. Interocular differences in higher-order aberrations on binocular visual performance. Optometry \& Vision Science 2008; 85(3): 174-179. DOI: 10.1097/OPX.0b013e31816445a7

[19] Lee SI, Yong JJ, Sohn H, et al. Effect of stimulus width on the perceived visual discomfort in viewing stereoscopic 3-D-TV. IEEE Transactions on Broadcasting 2013; 59(4): 580-590.

[20] Zhu M, Collins MJ, Robert ID. Microfluctuations of wavefront aberrations of the eye. Ophthalmic \& Physiological Optics the Journal of the British College of Ophthalmic Opticians 2004; 24(6): 562-71.

[21] Park S, Won MJ, Lee EC, et al. Evaluation of 3D cognitive fatigue using heart-brain synchronization. International Journal of Psychophysiology Official Journal of the International Organization of Psychophysiology 2015; 97(2): 120130. DOI: 10.1016/j.ijpsycho.2015.04.006

[22] Wenzel MA, Schultzekraft R, Meinecke FC, et al. EEG-based usability assessment of 3D shutter glasses. Journal of Neural Engineering 2015; 13(1): 016003. DOI: 10.1088/1741-2560/13/1/016003

[23] Hong X, Himebaugh N, Thibos LN. On-eye evaluation of optical performance of rigid and soft contact lenses. Optometry \& Vision Science Official Publication of the American Academy of Optometry 2001; 78(12): 872-80.

[24] Kim DS, Krassin J, Narváez J, et al. Comparison of the VISX wavescan and NIDEK OPD-scan aberrometers. Journal of Refractive Surgery 2009; 25(5): 429-434. DOI: 10.3928/1081597X-20090422-05

[25] Yong L. Ocular higher-order aberrations and visual performance. Durham University 2011; (in Chinese). 